\title{
Micelle self-assembly and chain exchange kinetics of tadpole block copolymers with a cyclic corona block
}

Ammu Prhashanna, Elena E. Dormidontova*

Polymer Program, Institute of Materials Science and Physics Department, University of Connecticut, Storrs, Connecticut 06269, USA

*Corresponding Author E-mail: elena@uconn.edu

\section{Calculation of asphericity parameter}

The asphericity parameter $(\propto)$ characterizes particle shape and is defined as: ${ }^{1}$

$$
\propto=1-3 \frac{\left\langle E_{2}\right\rangle}{\left\langle E_{1}^{2}\right\rangle} ; \quad E_{1}=R_{1}+R_{2}+R_{3} ; \quad E_{2}=R_{1} R_{2}+R_{2} R_{3}+R_{1} R_{3}
$$

where $R_{1}, R_{2}$ and $R_{3}$ are the eigenvalues of the gyration tensor in decreasing order. The asphericity parmater ranges from 0 indicating a perfect sphere to 1 representing a very long rod. In Table S1, the average values of asphericity parameter for pure linear (L), pure tadpole (T) and mixed (LT20) micelles are reported. The distribution is calculated using a bin size of 0.1 . The ensemble average for $E_{1}$ and $E_{2}$ values are calculated using configurations over 10,000 time steps.

Table S1. Average asphericity values for different micelles system

\begin{tabular}{|c|c|}
\hline & $<\propto>$ \\
\hline $\mathrm{L}$ & $0.021 \pm 0.004$ \\
\hline $\mathrm{T}$ & $0.029 \pm 0.005$ \\
\hline LT20 & $0.025 \pm 0.005$ \\
\hline
\end{tabular}

\section{Calculation of diffusion coefficient}

To calculate the diffusion coefficient of copolymer chains, we simulate a single block copolymer chain in a cubic box of $10 r_{c}$ filled with solvent beads. The diffusion coefficient is calculated using 340,000 different configurations stored every $t=4.0$ time units. From these configurations we obtain the mean square displacement (MSD, Figure S1) of the $1^{\text {st }}$ bead in the chain with time and the average diffusion coefficient is calculated as:

$$
D=\frac{1}{6} \lim _{t \rightarrow \infty} \frac{\left\langle\left[r\left(t_{0}+t\right)-r\left(t_{0}\right)\right]^{2}\right\rangle}{t}
$$

where $r$ is the position vector, $t_{0}$ is initial time, $t$ is at time $t$ and $\mathrm{D}$ is the diffusion coefficient. The term $\left[r\left(t_{0}+t\right)-r\left(t_{0}\right)\right]^{2}$ is defined as the mean square displacement (MSD) and the slope is used to compute the apparent diffusion constant (see main text). 


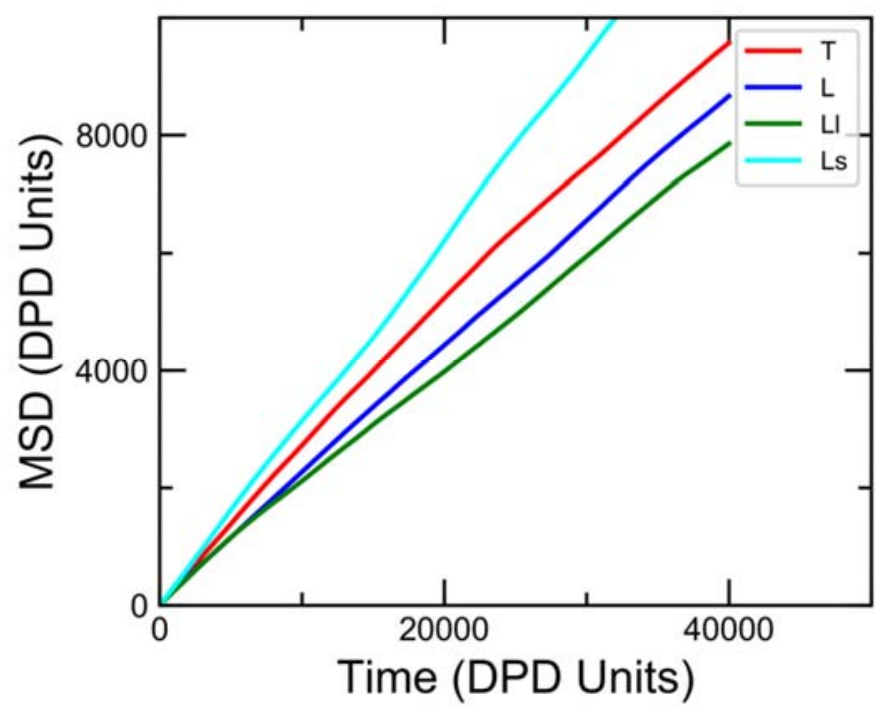

Figure S1. Mean square displacement of linear (L), tadpole (T), short linear (Ls) and long linear (Ll) chains in solvent.

\section{Chain expulsion function $(\mathrm{E}(\mathrm{t}))$}

At any time, $t=0$, all polymer chains within the micelle are labelled and are tracked with time. Whenever the polymer chain leaves the micelle and remains as a free chain for 4 consecutive time frames (16.0 time units), then chain is unlabelled. The time evolution of the number of labelled chains $N(t)$ is monitored. The chain expulsion function is defined as: $E(t)=\langle N(t) / N(0)\rangle$, where $N(0)$ is the number of labelled chains at initial time, $t=0$ and $\langle\ldots\rangle$ denotes averaging over different initial states (see Figure S2).

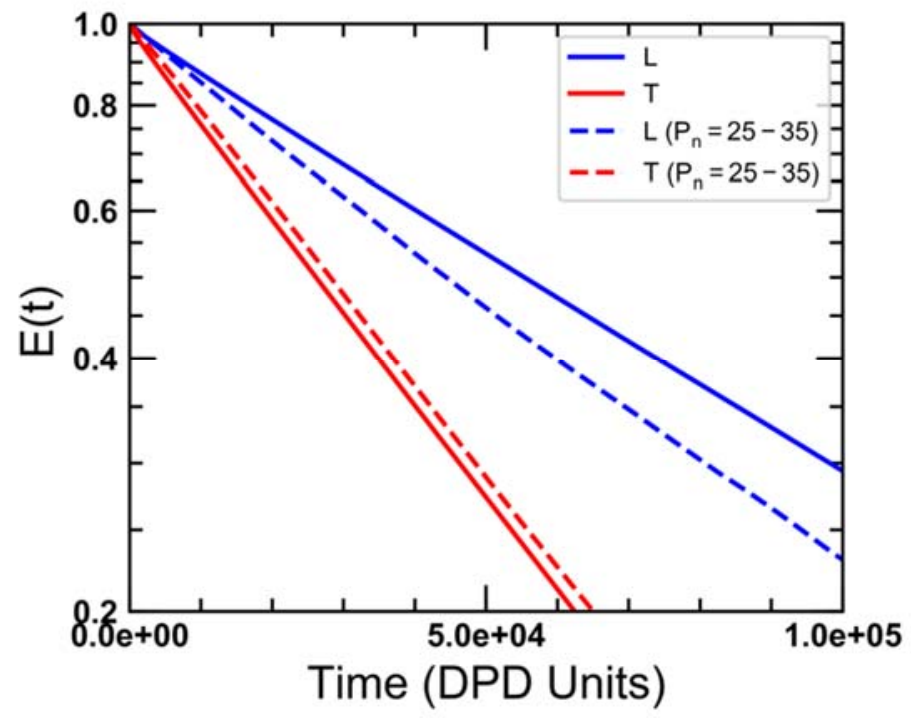


Figure S2. Chain expulsion function $(\mathrm{E}(\mathrm{t})$ ) for pure linear (solid blue) and pure tadpole (solid red) micelle systems, respectively. Dashed blue and dashed red lines indicate $\mathrm{E}(\mathrm{t})$ for pure linear and pure tadpole micelles with aggregation numbers between 25 and 35, which include the average aggregate number for tadpole micelles, 28.

\section{$\underline{\text { Interface of mixed micelles }}$}

The mixed micelle interface is characterized by analyzing the location of the corecorona junction point of linear and tadpole copolymers. The junction point is defined as the position of $1^{\text {st }}$ hydrophilic bead from the center of mass of the micelle. The core-corona junction point distributions are rather similar for both diblock and slightly shifted towards the corona for tadpole copolymers (i.e. chains slightly pulled out of the micelle core) compared to linear copolymers (Figure S3).

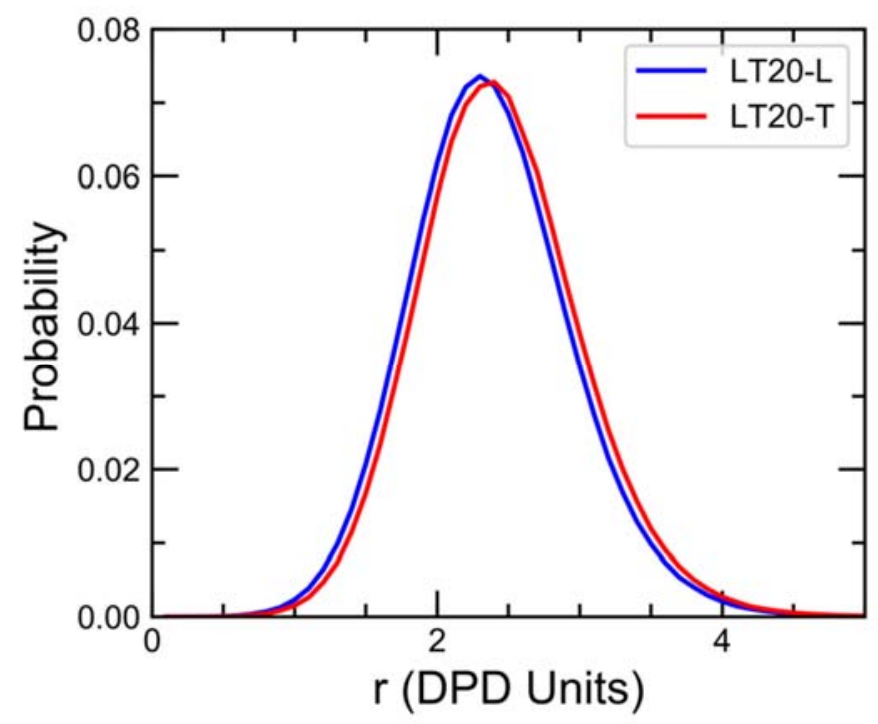

Figure S3. Probability distribution of junction point (1 st bead of corona block near core-corona interface) from the center of mass of micelle in LT20 system.

Micelles formed by linear diblock copolymer with shorter corona block, Ls

A diblock copolymer with a shorter hydrophilic block $A_{5} B_{6}(\mathrm{Ls})$ forms pure micelles of a slightly larger size (with an average aggregation number $P_{n}=44 \pm 4$, Figure S4) that exhibit rather similar chain exchange kinetics to the $A_{5} B_{8}$ copolymer (L) based on the overall contrast function $\mathrm{C}(\mathrm{t})$ (Figure $\mathrm{S} 5)$. 


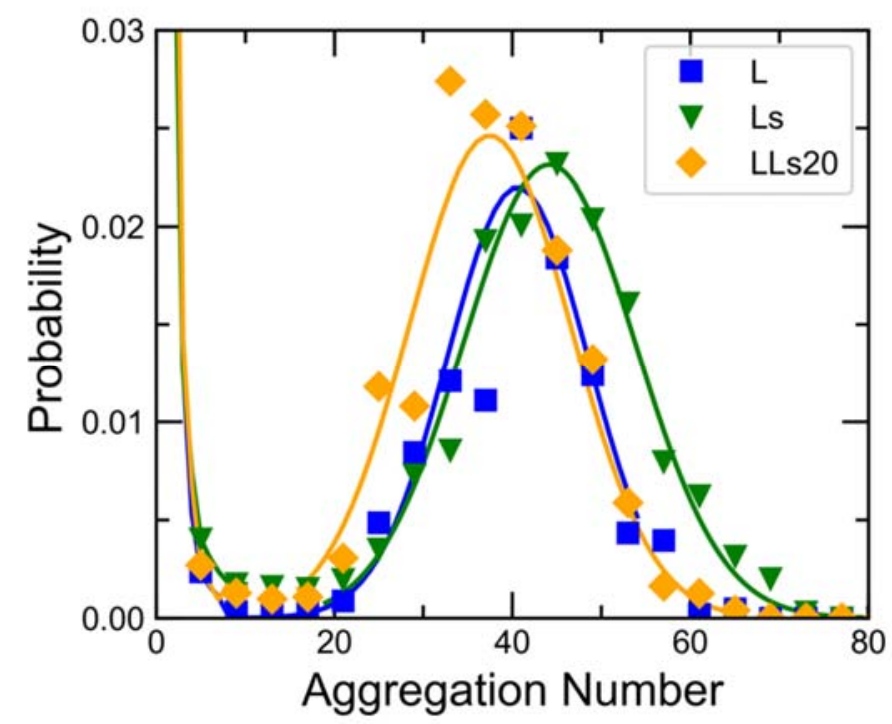

Figure S4. Aggregation number distribution for micelles formed by $A_{5} B_{8}$ linear diblock (L, blue squares), $A_{5} B_{6}$ short linear diblock (Ls, green triangles) and mixed micelles formed by 20\% short linear: $80 \%$ linear chains (LLs20, orange diamonds)

We find that the mixed LLs20 micelles $\left(P_{n}=37 \pm 4\right)$ exchanged chains in a very similar manner for pure and mixed micelles with no synergistic effect observed (Figure S5) even for chain escape, (Figure S6).

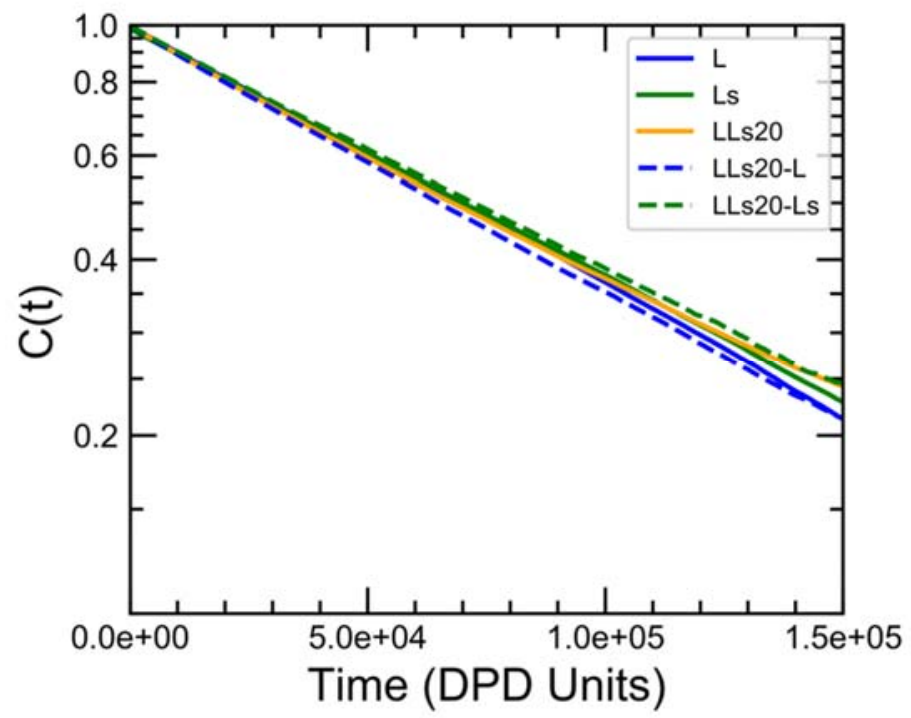

Figure S5. Contrast function of linear and short linear chains in pure linear (L), pure short linear (Ls) and mixed (LLs20) micelles. 


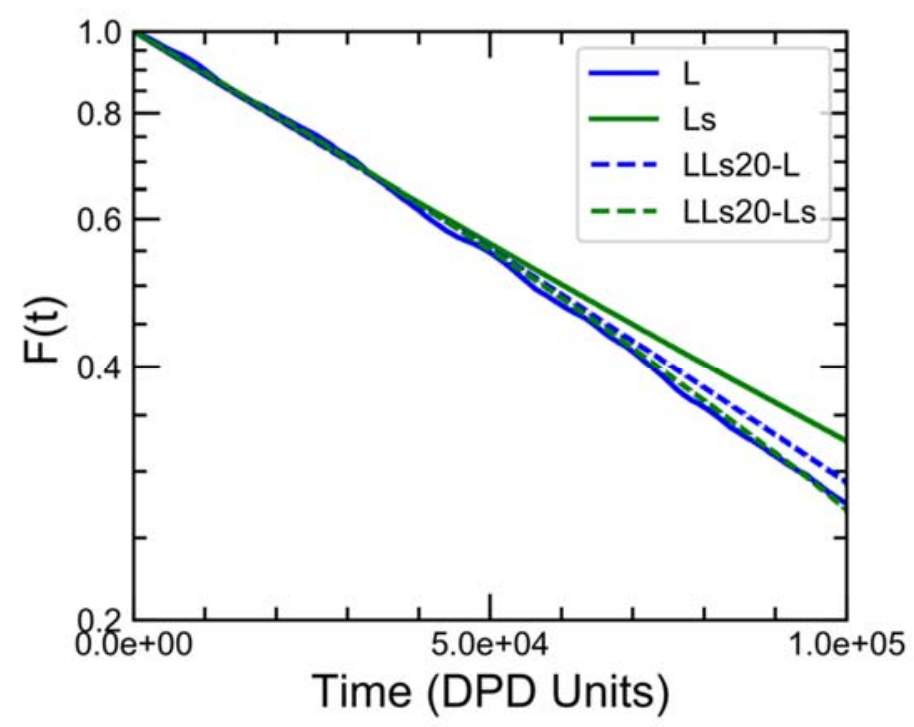

Figure S6. Native chain expulsion function for linear and short linear unimers for pure linear (L), pure short linear (Ls) and mixed (LLs20) micelles with aggregation number $30 \leq P_{n} \leq$ 50.

Table S2. An average micelle aggregation number $P_{n}$, core size of the micelle $\left(R_{C}\right.$ in DPD units) and area per chain at the core-corona interface in L, Ls, and LLs20 micelle systems

\begin{tabular}{cccc}
\hline & $P_{n}$ & $R_{C}$ (DPD units) & Area/chain \\
\hline $\mathrm{L}$ & $41 \pm 4$ & 2.34 & $1.67 \pm 0.16$ \\
\hline $\mathrm{Ls}$ & $44 \pm 4$ & 2.46 & $1.73 \pm 0.16$ \\
\hline LLs20 & $37 \pm 4$ & 2.27 & $1.75 \pm 0.19$ \\
\hline
\end{tabular}

Table S3. Fraction of copolymer chains in small aggregates $\left(2 \leq P_{n} \leq 10\right)$ and unimers for $\mathrm{L}$, Ls, and LLs20 micelle systems.

\begin{tabular}{ccc}
\hline & L & Ls \\
\hline L & $0.026 \pm 0.014$ & \\
\hline Ls & & $0.016 \pm 0.013$ \\
\hline LLs20 & $0.030 \pm 0.011$ & $0.024 \pm 0.011$ \\
\hline
\end{tabular}

\section{References:}

1. Sheng, Y.-J.; Wang, T.-Y.; Chen, W. M.; Tsao, H.-K. A- B diblock copolymer micelles: Effects of soluble-block length and component compatibility. The Journal of Physical Chemistry B 2007, 111 (37), 10938-10945. 